\title{
Ante Sikirica
}

E-mail: asikirica@riteh.hr

University of Rijeka, Center for Advanced Computing and Modelling

Radmile Matejčić 2, 51000 Rijeka, Croatia

\section{Ivana Lučin}

E-mail: ilucin@riteh.hr

\section{Zoran Čarija}

E-mail: zcarija@riteh.hr

\section{Bože Lučin}

E-mail: blucin@riteh.hr

University of Rijeka, Faculty of Engineering

Vukovarska 58, 51000 Rijeka, Croatia

\section{CFD Analysis of Marine Propeller Configurations in Cavitating Conditions}

\begin{abstract}
Diversely performing propellers as a consequence of design variability are nowadays a commonplace. Fundamental geometric particularities, including size, stipulate performance characteristics, which are usually the only required parameters when deciding on a propeller for specific purpose. With the main focus on the performance, accompanying phenomena, e.g. cavitation, tend to be overlooked. In this paper, propeller configurations in cavitating flow are investigated, with emphasis on real-world performance differences caused by cavitation. Recommended CFD approach is presented with respect to configuration specifics. Available experimental data is used as a baseline for a single propeller, which is then analysed in ducted and tandem configurations with resulting cavitation extents and shape evaluated in the context of current designs.
\end{abstract}

Keywords: CFD, cavitation, propeller, ducted, tandem 


\section{Introduction}

Cavitation as a phenomenon is usually unavoidable in real-world circumstances especially in rotating and vibrating machinery. Turbomachinery and marine engineering are thus fields most commonly invested into analysis, prediction and mitigation of cavitation, which occurs as a consequence of pressure drops. When the local pressure drops below vapor saturation pressure, vapor bubbles (pockets) are formed. These bubbles move with the stream until their eventual implosion. From a strictly structural standpoint, cavitation causes erosion and corrosion of machinery, which eventually leads to the deterioration of a mechanical part in question. Apart from physical damage, cavitation can cause noise and vibrations and in general drives performance downturn.

Commonly, cavitation is analysed experimentally due to somewhat lacking numerical models that describe it. However, experimental tests conducted in cavitation tunnels are done in controlled environments and are limited by the tunnel characteristics. Traditional numerical methods, though convenient, tend to oversimplify the problem and are thus being phased out by CFD, which in conjunction with the experimental baseline, can provide adequate cavitation predictions, at least in the context of performance assessment.

Pereira et al. [1] conducted experimental measurements and numerical analysis of a propeller in cavitating conditions using Boundary Element Method (BEM). Sheet cavitation extents for the analysed E779A propeller were predicted with relative accuracy, however, additional cavitation types were not observed. Bosschers et al. [2] introduced a system which integrates Reynolds-averaged Navier Stokes (RANS) equations for viscous flow analysis and BEM for cavitating and acoustics analyses. Validation was done on several propeller designs, however, cavitation predictions, considering they are based on BEM, attested to the same shortcomings when predicting cavitation types. In their study, Bensow and Bark [3] analysed and proposed the use of Large Eddy Simulations (LES) for cavitation analyses, at minimum as a validation of the final design. They used implicit LES scheme along with Kunz mass transfer model to validate their stance. Results for open water and cavitation conditions were within $3 \%$ of the experimental values for analysed advance ratios. Performance characteristics where thus adequately predicted, however, cavitation extent predictions, though acceptable, showed discrepancies. More importantly, it was concluded, based on conducted and previous studies, that the choice of mass transfer model has negligible or no impact on cavitation prediction, whereas spatial and temporal discretization along with solver type, could influence the results significantly. Salvatore et al. [4] presented unified results for the E779A propeller in different conditions and for various methodologies. Open water results were comparable to experimental measurements, whereas cavitation predictions, though numerically acceptable, depending on the methodology and solver in use, exhibited various degrees of discrepancy in cavity extent predictions. Thus, a definitive approach for cavitation analysis could not be suggested. Further cavitation investigations of E779A were conducted by [5], [6] and 
[7]. $k-k_{L}-\omega, k-\varepsilon$ and RNG $k-\varepsilon$ turbulence models were used respectively, with acceptable and overall similar results to [4].

Aforementioned studies focused on traditional, single propeller designs. Though simple, they perform competently and most importantly, from a manufacturing standpoint, are easy and cheap to manufacture. These choices impose certain restrictions, both on performance and cavitation susceptibility. Ducted propeller configurations offer several benefits when compared to traditional propeller designs. Benefits are varying, but limited, and for higher advance ratios can be negligible. Thus, ducted propellers are considered as a situational alternative, mainly for lower speeds and higher loads or when cavitation is considered an issue. Consequently, ducts are classified as accelerating or decelerating ducts; accelerating ducts allow for increased efficiency while decelerating ducts cause increased static pressure thus postponing cavitation onset. Aside from ducted propellers, tandem configurations are also used as an alternative, though rarely in marine engineering.

Sanchez et al. [8] tried to validate CFD open water performance predictions for ducted propellers using RANS. Simulations were conducted on a Ka series propeller using MARIN 19A nozzle. $k-\varepsilon$ turbulence model was used to obtain steady-state results. Numerical errors were up to $20 \%$ when predicting performance characteristics, while the velocity distribution in the wake of the propeller was in relative accordance with available experimental data. Further work by Sanchez et al. [9] employed Multiple Reference Frame (MRF) method to analyze viscous flow around a ducted propeller with rudder, in order to ascertain the applicability of RANS for ducted propeller analysis in the context of recent CFD advancements. Authors emphasized the significance of proper grid generation since lacking interface zones between fluid regions in ducted propellers can lead to questionable results. Analysis was primarily done for open water conditions, however, regions with low pressure were identified so as to predict possible cavitation zones. Ducted configurations in cavitating conditions were analysed by Haimov et al. [10]. Obtained RANS results show potential, and depending on the complexity of the propeller, are relatively accurate in predicting cavitation, especially when compared to traditional inviscid tools. Further investigations were done by [11] and [12] emphasizing the influence of both ducts and cavitation on resulting propeller performance.

In this study, cavitation onset and consequent performance impact will be analysed. Established propeller test model - Potsdam Propeller Test Case (PPTC) is used as a baseline to validate our investigative approach. Cavitation analyses on PPTC are conducted in single, tandem and ducted configurations. Further tests are carried out on a custom propeller design in equivalent configurations so as to emphasize adverse effects cavitation can have, both structurally and in terms of overall performance, regardless of the configuration. 


\section{Methodology}

\subsection{Models and grids}

Throughout the course of this study, two propellers, namely PPTC VP1304 and custom designed V2, are used in various configurations both in open water and cavitating conditions. VP1304 is a five-bladed controllable pitch propeller. V2 is a simplified four-bladed fixed propeller, a design inspired by Wageningen series propellers and common consumer propellers. Overview of the geometric characteristics is given in Table 1 .

Table 1: Geometric characteristics of the propellers used in the study

\begin{tabular}{ccc}
\hline Model & VP1304 & V2 \\
\hline No. blades & 5 & 4 \\
Diameter $/ \mathrm{m}$ & 0.25 & 0.18 \\
P/D ratio & 1.635 & 1.127 \\
Hub ratio & 0.300 & 0.282 \\
\hline
\end{tabular}

Evaluated tandem propeller configurations are created using two equally oriented propellers positioned at a distance equal to the radius of the propeller in question. Rotational speeds of components in these designs are identical, thus propellers move in co-rotating fashion.

Ducted configurations employ similarly designed but differently sized ducts. Accelerating ducts use NACA 4415 profile as a template, whilst NACA 6412 serves as a basis for decelerating duct design. Geometric specifics of designed ducts are given in Table 2.

Table 2: Geometric characteristics of ducts used in the study

\begin{tabular}{lccrr}
\hline \multicolumn{1}{c}{ Model } & VP1304 acc. & VP1304 dec. & V2 acc. & V2 dec. \\
\hline$D_{\text {inlet }} / \mathrm{m}$ & 0.293 & 0.283 & 0.249 & 0.239 \\
$D_{\text {outlet }} / \mathrm{m}$ & 0.253 & 0.237 & 0.196 & 0.173 \\
Length $/ \mathrm{m}$ & 0.146 & 0.098 & 0.095 & 0.095 \\
\hline
\end{tabular}

In total, 8 different configurations have been envisioned. For each design, hybrid grids are created. Grid around the propeller is hexahedral structured while the rest of domain is unstructured. First cell height is chosen to ensure dimensionless wall distance $\mathrm{y}^{+} \sim 50$. An overview of created and meshed models is given in Picture 1. 

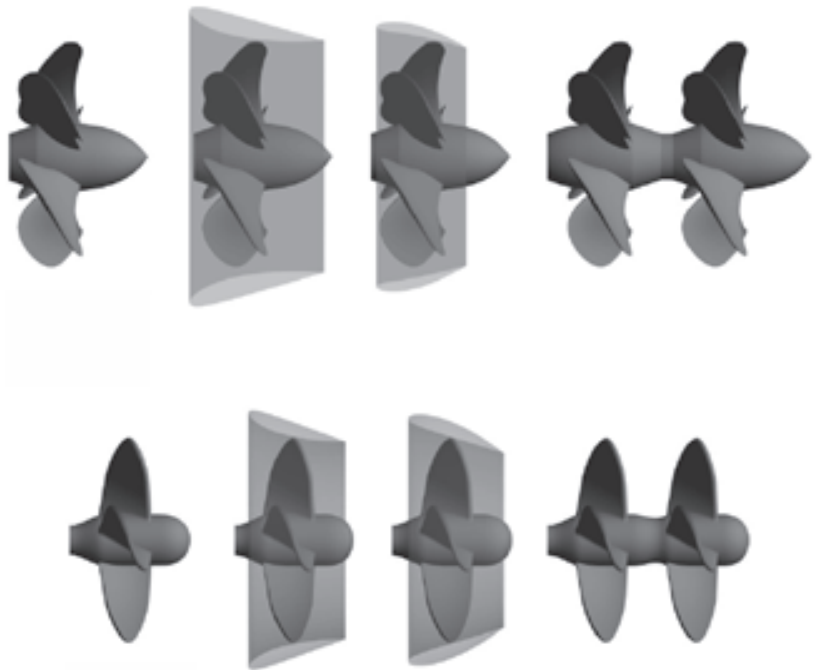

Figure 1: Configurations used in simulations: top VP1304, bottom V2; from left to right: single propeller, propeller with accelerating duct, propeller with decelerating duct, tandem propeller

\subsection{Numerical setup and hydrodynamic theory}

Computational domain has a cylindrical shape and spans $3 D$ in upstream and $7 D$ in downstream direction, where $D$ represents the diameter of the propeller. Crosssectional diameter is $5 D$. Domain size has been maintained across all tests. In order to reduce computational time, a single periodic passage has been analysed; these subdomains (Figure 2) correspond to one-fifth and one-fourth of the cylindrical domain respectively.

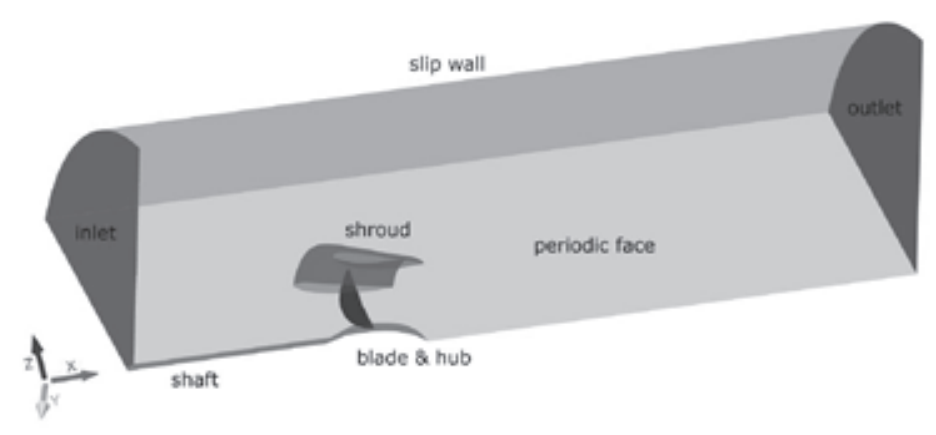

Figure 2: Domain and prescribed boundary conditions, VP1304 
On the outer surface of the computational domain, free-slip boundary conditions is set, while remaining walls have no-slip condition imposed. The inlet boundary has uniform velocity assigned. On the outlet, static pressure is set. MRF approach is adopted in the context of conducted steady-state simulations. Analyses are done using segregated solver with SIMPLE algorithm coupling pressure and velocity fields. Two equation $k-\varepsilon$ turbulence model is employed to close Navier-Stokes equations - model Reynolds stresses. Second order discretization schemes are used in all tests. Convergence is assumed if all residuals fall below $10^{-5}$ or torque and thrust show variance less than $0.1 \%$ during $10^{3}$ consecutive iterations. For cavitation tests, cavitation number $\sigma_{n}$ is ascertained from the local pressure with fixed fluid density, rotational speed and saturation pressure.

Relative performance of propellers is commonly expressed using dimensionless coefficients so as to facilitate comparison between different models. Values are given by means of diagrams, thus enabling quick comparison and selection of the proper model for a given task. The advance ratio represents the ratio between the advance speed $V_{A}$ and propeller tip speed $n D$ :

$$
J=\frac{V_{A}}{n D}
$$

where $n$ is the rotational speed in $\mathrm{s}^{-1}$, D diameter of the propeller in $\mathrm{m}$ and $V_{A}$ inflow velocity in $\mathrm{m} / \mathrm{s}$. Thrust and torque are issued as non-dimensional thrust and torque coefficients:

$$
\begin{aligned}
& K_{T}=\frac{T}{\rho n^{2} D^{4}} \\
& K_{Q}=\frac{Q}{\rho n^{2} D^{5}}
\end{aligned}
$$

where $T$ is the thrust in $\mathrm{N}, Q$ torque in $\mathrm{Nm}$ and $\rho$ density of the fluid in $\mathrm{kgm}^{-3}$. Finally, efficiency can be calculated as:

$$
\eta_{0}=\frac{J}{2 \pi} \frac{K_{T}}{K_{Q}} .
$$

\subsection{Validation}

Validation of previously described methodology has been done for both open water and cavitating conditions. Available experimental data on hydrodynamic characteristics of the VP1304 published by the SVA Potsdam [13] is compared to numerically attained values. Errors $\Delta K_{t}, \Delta 10 K_{q}$ and $\Delta \eta$ are calculated as relative errors with respect to the experimental data. Differences between experimental measurements and numerical 
simulations for open water tests are given in Table 3. Open water tests are done for three inflow velocities with the rotational speed set at $n=25 \mathrm{~s}^{-1}$. Error in torque coefficient prediction is consistently below $0.5 \%$, while thrust coefficient and efficiency differ at worst by $2.1 \%$ from experimental values.

Table 3: Open water validation, VP1304

\begin{tabular}{cccc}
\hline$V A / \mathrm{ms}-1$ & 3.583 & 4.917 & 6.262 \\
\hline$\Delta K_{t} / \%$ & 1.541 & 0.949 & 2.079 \\
$\Delta 10 K_{q} / \%$ & 0.418 & 0.376 & 0.323 \\
$\Delta \eta / \%$ & 1.127 & 1.320 & 1.762 \\
\hline
\end{tabular}

Validation in cavitating condition has been done for $V_{A}=6.367 \mathrm{~m} / \mathrm{s}(J=1.019)$ with equivalent rotational speed and cavitation number $\sigma_{n}=2.024$. For all hydrodynamic characteristics, predicted values differ less than $0.5 \%$ from experimental data. Numerical discrepancies between the experiment [13] and simulations for cavitating test are shown in Table 4.

Table 4: Cavitation validation, VP1304

\begin{tabular}{cc}
\hline$V A / \mathrm{ms}^{-1}$ & 6.367 \\
\hline$\Delta K_{t} / \%$ & 0.331 \\
$\Delta 10 K_{q} / \%$ & 0.073 \\
$\Delta \eta / \%$ & 0.404 \\
\hline
\end{tabular}

\section{Results and discussion}

\subsection{Open water results}

Open water curves for all configurations have been calculated for select ranges of advance ratios. Rotational speeds for VP1304 and V2 have been set at $n=25 \mathrm{~s}^{-1}$ and $n=30 \mathrm{~s}^{-1}$ respectively. Further tests on V2 have been conducted at $n=50 \mathrm{~s}^{-1}$. Results for initial tests are given in Figure 3. These results partially confirm well known facts; accelerating ducts can provide better efficiency while decelerating ducts usually have less of an impact on performance, with tandem co-rotating configurations performing the worst. It is shown that ducted configurations perform best at lower advance ratios, peaking approximately at the middle of the range, whilst at higher velocities their efficiency dips well below the results for a single propeller configuration, which is expected. This is not to say that benefits such as cavitation mitigation and noise reduction are mute, however, general performance is worse. 

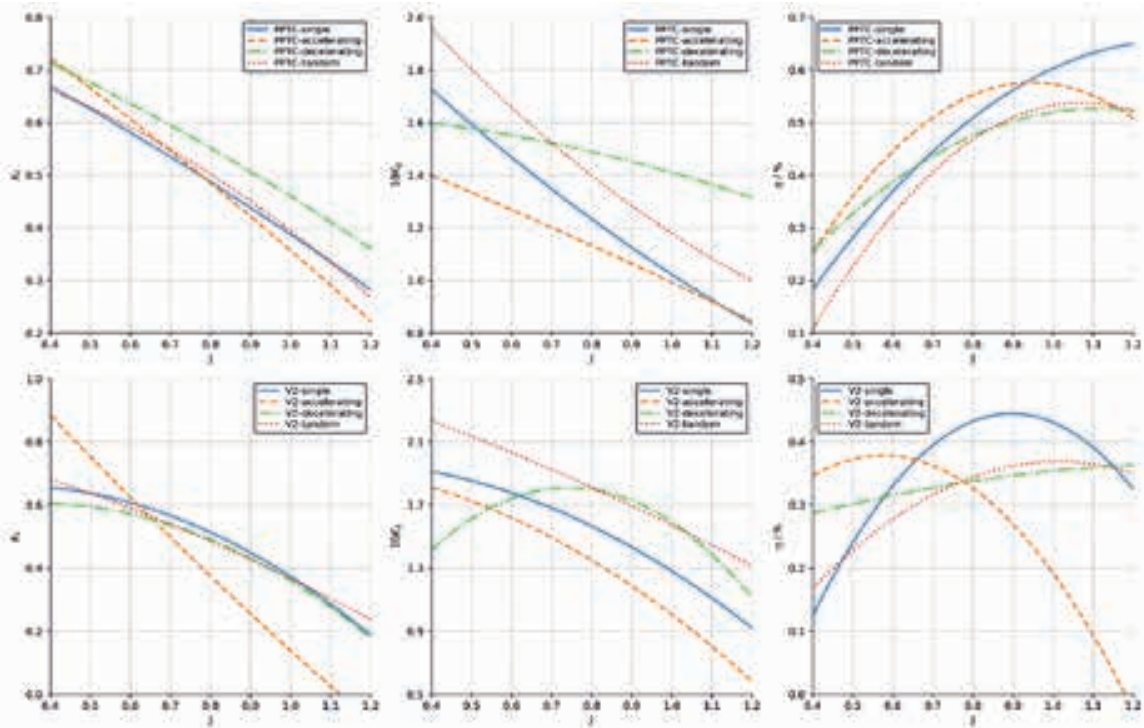

Figure 3: Open water results: top VP1304, bottom V2; from left to right: thrust coefficient $K_{t}$, torque coefficient $10 K_{q}$, efficiency $\eta$

\subsection{Cavitation results}

Cavitation tests on VP1304 are conducted for $\sigma_{n}=2.113$. Rotational speed has been set at $n=25 \mathrm{~s}^{-1}$ with saturation pressure $p_{v}=2873 \mathrm{~Pa}$. For the analysed advance ratio all configurations perform worse than single propeller model. Performance drop in an accelerating duct is the highest at around $13 \%$, while efficiency for a single propeller varied less than $0.5 \%$. An overview of efficiency variances is given in Table 5 .

Table 5: Cavitation driven efficiency drops, VP1304

\begin{tabular}{cc}
\hline Model & $\eta$ \\
\hline$\Delta \eta_{\text {single }} / \%$ & 0.433 \\
$\Delta \eta_{\text {accelerating }} / \%$ & 13.117 \\
$\Delta \eta_{\text {decelerating }} / \%$ & 1.283 \\
$\Delta \eta_{\text {tandem }} / \%$ & 6.720 \\
\hline
\end{tabular}


Cavitation extents shown in Figures 4 and 5 further muddle the results. Single propeller and tandem propeller results are expected, both in terms of performance impact and cavity extents. Pressure side tip and sheet cavitations are quite similar along with cavitation vortex rope. Aft blade in tandem configuration shows secondary sheet cavitation on suction side. This can be attributed to increased velocity in that region which drove static pressure down. Ducted configurations offer somewhat perplexing data. At lower advance ratios both configurations behave according to theory, however, with the inclusion of cavitation, benefits at higher speeds are negligible. It is evident that accelerating duct increases overall efficiency at lower speeds, however, it also has the greatest drop in performance. Curiously, cavity extent is limited mostly to blade tip region and hub, while decelerating duct shows marginally larger tip and sheet cavitation when compared to single propeller configurations. These results point to several important conclusions. At higher speeds, ducts produce higher drag than thrust, thus leading to lower performance. Cavitation mitigation depends not only on the configuration, but operating conditions as well, which complicates propeller choice. Finally, chosen duct configurations might not be able to fully capture the fundamentals behind ducts and their benefits, urging further investigations into adequate designs.

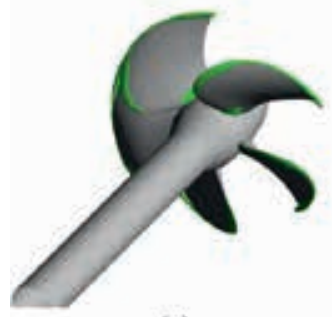

(a)

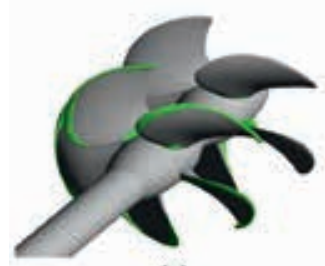

(c)

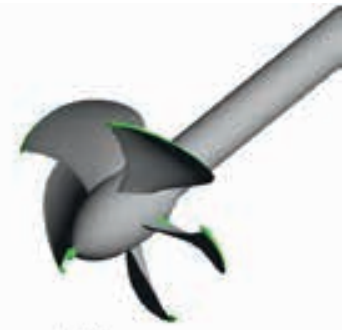

(b)

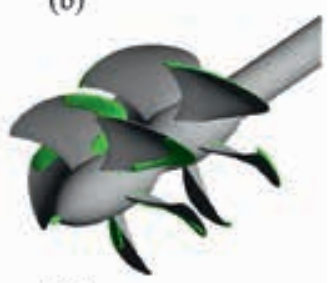

(d)

Figure 4: Cavity extents for $n=25 s^{-1}, J=1.019$ : VP1304 propeller, pressure side (a); VP1304 propeller, suction side (b); tandem VP1304 propellers, pressure side (c); tandem VP1304 propellers, suction side (d) 


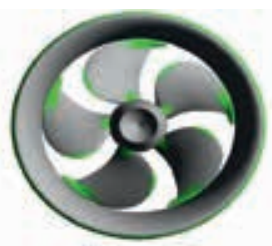

(a)

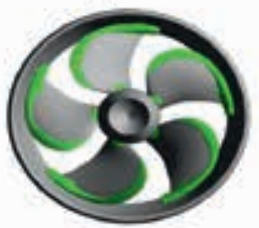

(c)

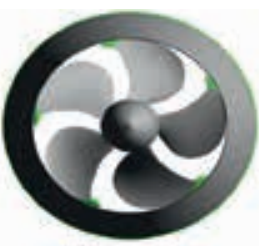

(b)

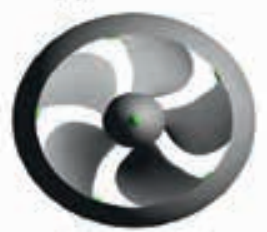

(d)

Figure 5: Cavity extents for $n=25 s^{-1}, J=1.019$ : VP1304 in accelerating duct, pressure side (a); VP1304 in accelerating duct, suction side (b); VP1304 in decelerating duct, pressure side (c); VP1304 in decelerating duct, suction side (d)

Further analyses have been done on a custom designed V2 propeller. V2 has no experimentally validated data, unlike VP1304, which has been thus tested at a typical advance ratio for said propeller. Therefore, two operating points with different rotational speeds are evaluated. Initially, rotational speed has been set at $n=30 \mathrm{~s}^{-1}$ with cavitation number $\sigma_{n}=4.956$ and saturation pressure $p_{v}=2873 \mathrm{~Pa}$. Inflow velocity and reference pressure have been set to $5 \mathrm{~m} / \mathrm{s}$ and $7.5 \cdot 10^{4} \mathrm{~Pa}$ respectively. Efficiency variances with respect to cavitation are given in Table 6 .

Table 6: Cavitation driven efficiency drops, $V 2, n=30 \mathrm{~s}^{-1}$

\begin{tabular}{cc}
\hline Model & $\eta$ \\
\hline$\Delta \eta_{\text {single }} / \%$ & 2.828 \\
$\Delta \eta_{\text {accelerating }} / \%$ & 14.448 \\
$\Delta \eta_{\text {decelerating }} / \%$ & 11.591 \\
$\Delta \eta_{\text {tandem }} / \%$ & 3.293 \\
\hline
\end{tabular}

Similarly to VP1304, configuration with accelerating duct has the largest efficiency drop, followed by decelerating duct configuration. Single propeller configuration is the most reliable, dropping in efficiency by less than $2 \%$. Figures 6 and 7 show the extent of cavitation for all configurations. Sheet cavitation is mostly absent. The leading edge is enveloped by cavitation in all test cases, including aft propeller in tandem configuration. Ducted configuration shows signs of tip vortex cavitation as well as hub cavitation. 


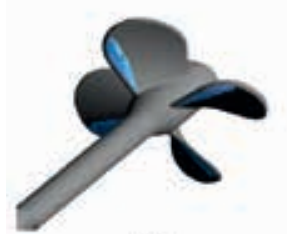

(a)

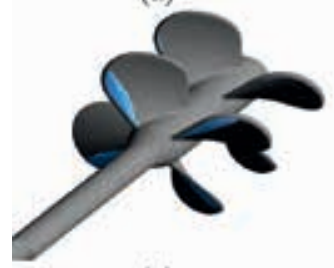

(c)

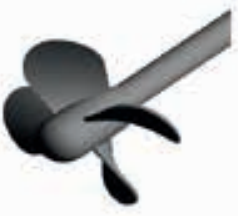

(b)

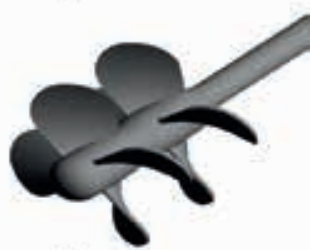

(d)

Figure 6: Cavity extents for $n=30 \mathrm{~s}^{-1}, J=0.926$ : V2 propeller, pressure side (a); $V 2$ propeller, suction side (b); tandem V2 propellers, pressure side (c); tandem V2 propellers, suction side (d)

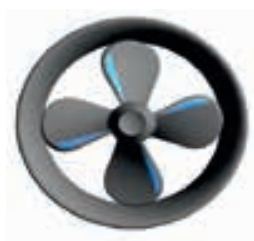

(a)

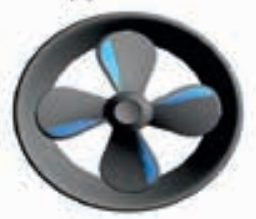

(c)

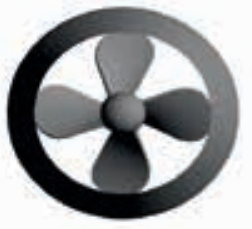

(b)

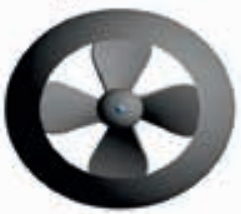

(d)

Figure 7: Cavity extents for $n=30 \mathrm{~s}^{-1}, J=0.926: \mathrm{V} 2$ in accelerating duct, pressure side (a); V2 in accelerating duct, suction side (b); V2 in decelerating duct, pressure side (c); V2 in decelerating duct, suction side (d)

With the increase in rotational speed, V2's risk of cavitation has grown significantly. For given cavitation number $\sigma_{n}=1.7841$ efficiency variances with respect to cavitation are shown in Table 7. 
Table 7: Cavitation driven efficiency drops, $V 2, n=50 \mathrm{~s}^{-1}$

\begin{tabular}{cc}
\hline Model & $\eta$ \\
\hline$\Delta \eta_{\text {single }} / \%$ & 1.882 \\
$\Delta \eta_{\text {accelerating }} / \%$ & 16.294 \\
$\Delta \eta_{\text {decelerating }} / \%$ & 13.377 \\
$\Delta \eta_{\text {tandem }} / \%$ & 3.580 \\
\hline
\end{tabular}

Efficiency drops are consistent with all previous tests. This further emphasizes the necessity of additional tests so as to determine whether these results are a consequence of numerical errors or in reality correspond to performance drops which are usually not noted in open water tests. Noted cavitation patterns for $n=50 \mathrm{~s}^{-1}$ are shown in Figures 8 and 9 . Resulting patterns are a direct consequence of forced cavitating condition. The root of the blade on suction side of a tandem propeller is exposed to sheet cavitation. Cavitation vortex rope and trailing edge cavitation show variance between ducted configuration, with decelerating model exhibiting larger cavity extents. Sheet cavitation is overall similar for all configurations.

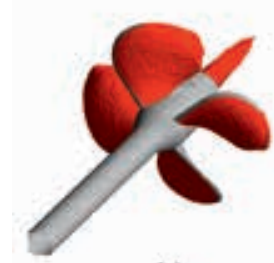

(a)

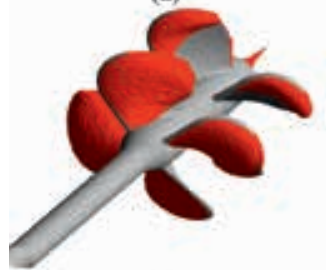

(c)

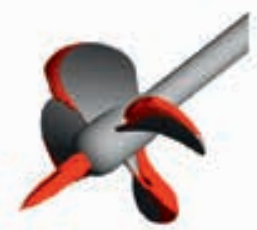

(b)

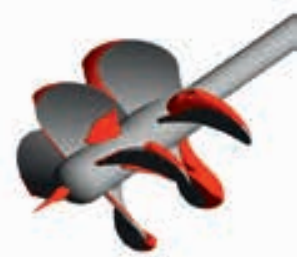

(d)

Figure 8: Cavity extents for $n=50 \mathrm{~s}^{-1}, J=0.556$ : V2 propeller, pressure side (a); $V 2$ propeller, suction side (b); tandem V2 propellers, pressure side (c); tandem V2 propellers, suction side (d) 


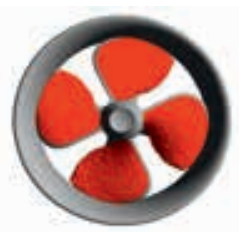

(a)

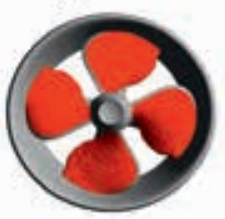

(c)

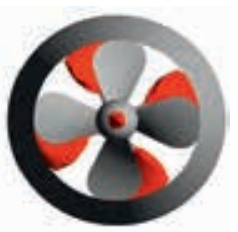

(b)

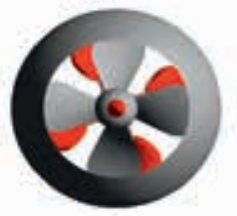

(d)

Figure 9: Cavity extents for $n=50 \mathrm{~s}^{-1}, J=0.556$ : $V 2$ in accelerating duct, pressure side (a); V2 in accelerating duct, suction side (b); V2 in decelerating duct, pressure side (c); V2 in decelerating duct, suction side (d)

\section{Conclusion}

Throughout the course of this study, multiple PPTC VP1304 designs have been analyzed in open-water and cavitating conditions using turbulence models. Validation study has been conducted to verify general approach. Encouraged by good agreement with experimental values (variances less than $2.1 \%$ ), custom propeller design has been included in the assessment. Single propeller, ducted and tandem configurations have been reviewed with respect to cavitation and efficiency drops. Accelerating duct for analyzed advance ratio shows greatest performance drop with smallest cavity extents. This phenomenon can be attributed to excessive drag caused by cavitation regions on the nozzle. Other configurations show similar behavior across all tests. On average, single propeller configuration seems to drop the least in efficiency, which is not uncommon. It is a well-known fact that for higher inflow velocities ducted configurations offer no significant benefits. Performance drops should be further investigated with the inclusions of a grid sensitivity study in order to verify the validity of results for ducted configurations; performance drops could be a result of numerical errors. Reduction/increase in cavity extents depends not only on the configuration, but operating conditions. Consequently, choice of a proper configuration is situational. Since this study used arbitrary duct designs, obtained results might not have fully captured benefits of ducts in marine engineering. It is evident that the use of arbitrarily designed nozzles is not the most appropriate and prompts further analyses and eventually design optimization. 


\section{References}

1. Pereira, F., Salvatore, F. and Di Felice, F., "Measurement and modeling of propeller cavitation in uniform inflow," Journal of Fluids Engineering, vol. 126, no. 4, pp. 671-679, 2004.

2. Bosschers, J., Vaz, G., Starke, A. and van Wijngaarden, E., "Computational analysis of propeller sheet cavitation and propeller-ship interaction," in Proceedings of the RINA Conference "MARINE CFD2008”, Southampton, UK, pp. 26-27, 2008.

3. Bensow, R. E. and Bark, G., "Implicit les predictions of the cavitating flow on a propeller," Journal of fluids engineering, vol. 132, no. 4, p. 041302, 2010.

4. Salvatore, F., Streckwall, H. and Van Terwisga, T., "Propeller cavitation modelling by cfd-results from the virtue 2008 rome workshop," in Proceedings of the First International Symposium on Marine Propulsors, Trondheim, Norway, pp. 22-24, Citeseer, 2009.

5. Fujiyama, K. and Hitomi, D., "Performance and cavitation evaluation of marine propeller using numerical simulations," in Second International Symposium on Marine Propulsors, SMP, vol. 11, 2011.

6. Subhas, S., Saji, V., Ramakrishna, S. and Das, H., "Cfd analysis of a propeller flow and cavitation," International Journal of Computer Applications, vol. 55, no. 16, 2012.

7. Zhu, Z.-f. and Fang, S.-1., "Numerical investigation of cavitation performance of ship propellers," Journal of Hydrodynamics, Ser. B, vol. 24, no. 3, pp. 347-353, 2012.

8. Sanchez-Caja, A., Rautaheimo, P. and Siikonen, T., "Simulation of incompressible viscous flow around a ducted propeller using a rans equation solver," in Proceedings of the Twenty-Third Symposium on Naval Hydrodynamics, 2000.

9. Sánchez-Caja, A., Pylkkänen, J. and Sipilä, T. P., "Simulation of the incompressible viscous flow around ducted propellers with rudders using a ranse solver," in 27th Symposium on Naval Hydrodynamics, 2008.

10. Haimov, H., Bobo, M. J., Vicario, J. and Del Corral, J., "Ducted propellers. a solution for better propulsion of ships. calculations and practice," in First International Symposium on Fishing Vessel Energy Efficiency E-Fishing, 2010.

11. Bertetta, D., Bertoglio, C., Conti, F., Rizzo, C. M. and Viviani, M., "Cavitation tunnel tests on ducted propellers," in Proceedings 17th International Conference on Ships and Shipping Research, 2012.

12. Gaggero, S., Rizzo, C., Tani, G. and Viviani, M., "Design, analysis and experimental characterization of a propeller in decelerating duct," in Third International Symposium on Marine Propulsors (SMP2013), Launceston, Tasmania, Australia, 2013.

13. SVA Team, "PPTC smp'11 Workshop." https://www.sva-potsdam.de/ pptc-smp11-workshop/, 2011. Accessed: 7-May-2019. 